\title{
Allogeneic hematopoietic cell transplantation in mantle cell lymphoma
}

\author{
Ryan D. Cassaday, MD and Ajay K. Gopal, MD \\ Clinical Research Division, Fred Hutchinson Cancer Research Center; Division of Medical \\ Oncology, Department of Medicine, University of Washington; Seattle, WA
}

\begin{abstract}
Mantle cell lymphoma (MCL) is a high-risk non-Hodgkin lymphoma that is considered incurable with standard chemotherapy. While autologous hematopoietic cell transplantation (autoHCT) can provide lengthy disease-free survival in select patients, cure generally is not an expected outcome with this approach. Allogeneic hematopoietic cell transplantation (alloHCT), which can exploit the potential benefits of graft-versus-lymphoma (GVL) effect, has been shown in multiple studies to yield a small but reproducible portion of patients with long-term remission more suggestive of cure. Historically, alloHCT for MCL was administered after myeloablative conditioning, but this approach was limited by early non-relapse mortality. Development of reduced-intensity (RI)alloHCT has abrogated some of the early post-transplant risks, allowing this potentially effective therapy to be offered to a larger number of affected individuals. The trends in published data reflect a preference toward using RI-alloHCT for MCL, often because patients in whom alloHCT is being considered have relapsed disease following myeloablative autoHCT. Further efforts to spare the effects of graft- versus-host disease (GVHD) while still evoking GVL remain a focus of investigation in this area. In this review, we will discuss the application of alloHCT in the management of MCL, the factors associated with outcome, the different methods in which it can be performed, and the strategies that can be employed in post-alloHCT relapse.
\end{abstract}

\section{Keywords}

mantle cell lymphoma; allogeneic transplantation

\section{Introduction}

Despite improvements in outcome with chemotherapy, mantle cell lymphoma (MCL) is not considered curable with this approach. Strategies including more intense multi-agent chemotherapy as induction followed by consolidation with autologous hematopoietic cell transplantation (autoHCT) have demonstrated higher response rates, improved progressionfree survival, and the possibility of improved overall survival [1-3]. However, even with this aggressive approach, relapse frequently occurs, with the worst outcomes in those with the highest mantle cell international prognostic index (MIPI) scores [4, 5]. Once relapse occurs after autoHCT, survival can be limited, especially for those transplanted beyond first

\footnotetext{
(C) 2012 Elsevier Ltd. All rights reserved.

Corresponding Author: Ajay Gopal, MD, 1100 Fairview Ave. N., Mailstop G3200, Seattle, WA 98109, agopal@uw.edu, Telephone: (206)288-2037, Fax: (206)288-1130.

Publisher's Disclaimer: This is a PDF file of an unedited manuscript that has been accepted for publication. As a service to our customers we are providing this early version of the manuscript. The manuscript will undergo copyediting, typesetting, and review of the resulting proof before it is published in its final citable form. Please note that during the production process errors may be discovered which could affect the content, and all legal disclaimers that apply to the journal pertain.
} 
complete or partial remission or with relapses within 1 year of transplant [6]. Taken together, data to date suggest that though intensified initial approaches may improve outcomes in MCL, most patients will eventually experience relapse and succumb to their disease.

Allogeneic hematopoietic cell transplantation (alloHCT) is felt to be the only therapeutic strategy with curative potential. Unlike the dependence on the cytotoxic effects of chemotherapy in the approaches mentioned above, it is thought that a graft-versuslymphoma (GVL) effect provides an additional mechanism of activity against MCL [7]. More recently, the advent of reduced-intensity conditioning regimens has allowed this treatment to be administered safely to older patients, allowing its use in a greater number of patients. Widespread use of the approach remains limited by the availability of a suitably human leukocyte antigen (HLA)-matched stem cell source, as well as the potential toxicities, including graft-versus-host disease (GVHD). In this review, we will discuss the application of alloHCT in patients with MCL.

\section{Comparison of Autologous vs Allogeneic HCT in MCL}

AutoHCT is an important strategy used in MCL. According to the National Comprehensive Cancer Network (NCCN) guidelines, autoHCT should be considered to consolidate the first complete remission in patients eligible for this aggressive approach [8]. This same group recommends that alloHCT be used to consolidate the second complete remission in the event of relapse. As discussed above, autoHCT relies primarily on the cytotoxic effects of the conditioning regimen, while alloHCT includes both this effect as well as GVL, the potential for immune-mediated anti-lymphoma effects by the donor immune system. Though both strategies have their potential benefits and limitations, direct comparisons between them are relatively sparse in the literature. Moreover, because of the relative rarity of this diagnosis, the numbers present in these studies are usually too small to draw any broad conclusions, and they are often performed in a retrospective fashion. That said, certain themes and trends can be drawn from these efforts.

The earliest prospective study comparing auto- and alloHCT was published by Khouri and others at M.D. Anderson Cancer Center (MDACC) and also was one of the first published studies addressing the role of hematopoietic cell transplantation in mantle cell lymphoma [9]. This study evaluated patients that had received 4 cycles of the multi-agent chemotherapy regimen hyperCVAD (hyperfractionated cyclophosphamide, vincristine, doxorubicin and dexamethasone alternating with cycles of high-dose methotrexate and cytarabine). Following this, patients then went on to stem cell transplant: If the patient was less than 55 years of age and had an HLA-matched or single-antigen mismatched related donor, they received an alloHCT; if the patient was less than 65 years old and lacked an adequately HLA-matched related donor, they went on to receive an autoHCT. A total of 34 patients were able to be transplanted, with 26 receiving autoHCT and 8 receiving alloHCT. All patients achieved a complete remission (CR) following the 4 cycles of hyperCVAD and the conditioning regimen (which was cyclophosphamide and total body irradiation [TBI] in all cases). In the alloHCT group, 2 patients died from GVHD within 10 months, while the other 6 remained in a CR. This yielded an estimated 3-year overall and event-free survival for alloHCT of 73\%. This compares favorably to the autoHCT cohort, which had an eventfree survival (EFS) of $28 \%$ and overall survival (OS) of $48 \%$ at $3 \mathrm{yrs}$. While this difference was not statistically significant $(\mathrm{P}=0.29$ for $\mathrm{EFS} ; \mathrm{P}=0.83$ for $\mathrm{OS}$ ), it does suggest a longterm benefit for alloHCT if the associated toxicity from GVHD can be mitigated.

Another prospective study by Evens and colleagues tried a similar approach to compare auto-and alloHCT for MCL [10]. However, this study was different in that all patients were 
treatment-naïve. The induction chemotherapy regimen utilized a unique multi-agent approach consisting of CTAP (cyclophosphamide, teniposide, doxorubicin, and prednisone) alternating with VMAC (vincristine, high-dose methotrexate, and cytarabine). They used a similar "biological allocation" to determine who would receive autoHCT versus alloHCT, and all patients received a transplant conditioning regimen of busulfan and TBI. A total of 17 patients were able to be transplanted, with only 4 patients in the alloHCT group. Of these 4 patients, 1 died from chronic GVHD after 24 months, and 2 died from progressive disease (PD) at 22 and 29 months, respectively. The remaining patient was alive at 67 months in a continuous CR, yielding 5-year EFS and OS for alloHCT of 25\%. Of the 13 patients treated with autoHCT, the 5- year EFS was 54\% and OS was 75\%, while the treatment-related mortality (TRM) was $0 \%$. Again, because of the small numbers, no statistically significant differences could be gleaned from these data. They do show that long-term benefit is possible from both of these strategies when used early in the treatment of MCL.

The largest retrospective study that compares auto- and alloHCT also came from MDACC [11]. These data came from long-term follow-up of a risk-adapted transplantation strategy for MCL which spanned nearly 20 years. Generally, patients that responded well to induction chemotherapy were consolidated with autoHCT, whereas those patients with relapsed or primary refractory disease were given a reduced-intensity alloHCT if an HLAmatched sibling was available; if a matched sibling was not available, autoHCT was used. Because of the length of time over which these patients were accrued, there was substantial heterogeneity in the manner in which these patients were treated, particularly as it relates to their induction and conditioning regimens. With that caveat stated, perhaps not surprisingly, the group receiving autoHCT in first remission $(n=50)$ had superior progression-free (PFS) and OS compared to those receiving autoHCT later in their course $(n=36)$. However, when the patients with relapsed or primary refractory disease were compared, those who received alloHCT $(\mathrm{n}=35)$ had statistically better 6-year OS $(53 \%)$ and PFS $(46 \%)$ compared to those treated with autoHCT (35\% and $10 \%$ at 4 years, respectively; $\mathrm{P}=0.005$ for OS, $\mathrm{P}=0.01$ for PFS). These rates for alloHCT were not statistically significantly different than for those receiving autoHCT in first remission (61\% and 39\%, respectively). Interestingly, plateaus were seen in both the PFS and OS curves for the alloHCT group, with 9 patients (25\%) not having relapsed or died after a range of 63-110 months of follow-up, 3 of whom had previously received autoHCT; such patterns were not seen in the groups treated with autoHCT. For all groups, TRM was relatively similar, with rates at 12 months not exceeding $10 \%$ in any of these cohorts. Taken together, these data support the concept that alloHCT may offer the possibility for durable remission (perhaps even cure) for those with relapsed/ refractory disease that autoHCT may not provide. Despite this optimism, the rate of TRM seen from alloHCT in this study is lower than that reported by many other groups, so perhaps this study understates the potential toxicity from this approach.

As the above study highlights, alloHCT is often not used in first remission, instead implementing it for relapsed or refractory disease. Reserving alloHCT until later in the natural history of MCL means patients receiving this therapy often have a disease biology that is more refractory to conventional therapy. This concept is highlighted in a different series published from Johns Hopkins University [12]. In a cohort of 58 patients, median EFS following autoHCT was significantly longer than that for alloHCT (43 months versus 5.5 months, respectively; $\mathrm{P}=0.005$ ). However, there were a higher proportion of patients with relapsed disease that were treated with alloHCT. When limiting the analysis to those in first remission (either complete or partial; $\mathrm{n}=10$ for alloHCT, and $\mathrm{n}=26$ for autoHCT), the projected 3-year EFS was approximately $70 \%$ for both the autoHCT and alloHCT groups. Additionally, they noted that only 1 patient (10\%) treated with alloHCT in first remission had relapsed during the study period compared to 5 patients (19\%) who received autoHCT in first remission. The rate of TRM was 11\% in the autoHCT cohort compared to 53\% in the 
alloHCT group. Since all but one patient received similar myeloablative transplant conditioning regimens (cyclophosphamide plus either TBI or busulfan), the differences seen are most likely attributable to the effects and complications of an engrafted donor immune system. Similar results were seen in a smaller retrospective study from the University of Minnesota [13]. In this study, the majority of patients who received autoHCT $(n=13)$ were in first remission, whereas the majority of those treated with alloHCT $(n=17)$ had relapsed or refractory disease, including 3 patients that had relapsed following autoHCT. Despite this relative imbalance, the 5-year OS and PFS for autoHCT were $42 \%$ and $31 \%$, respectively, compared to $49 \%$ and 50\%, respectively, for alloHCT (not statistically different, P-value not reported). No relapses were seen beyond 1 year in the alloHCT group. This disease-specific survival was tempered by an increased rate of TRM, with 1-year rates for alloHCT of $29 \%$ compared to no treatment-related deaths from autoHCT. While limited by the small numbers, these data suggest that either transplantation approach can be effective when used early in the disease course, but transplant-related complications could make alloHCT a less attractive option at this stage.

\section{Strategies Using AlloHCT in MCL}

\section{Myeloablative versus Reduced-Intensity AlloHCT}

The initial applications of alloHCT in the management of MCL primarily utilized a myeloablative conditioning regimen [7,9]. This approach offers the same benefits as autoHCT in terms of intensity of therapy. With the exception of the retrospective study by Tam and colleagues from MDACC, the studies discussed in the previous section compared similar myeloablative conditioning regimens, with patient groups differing primarily by the presence of either an autologous or allogeneic stem cell source. The drawback to this approach is the inherent early morbidity and mortality from the intense chemotherapy and/or radiation included. For patients that are older or have significant medical comorbidities, myeloablative conditioning can be prohibitively toxic. Thus, reduced-intensity conditioning regimens were developed to include such patients within the potentially curative benefit of allografting $[14,15]$. These regimens tend to be fludarabine-based with the focus on providing sufficient immunosuppression to allow engraftment of the donor immune system, and primarily relying on the GVL effect to produce or maintain clinical responses.

Weighing the possible risks and benefits of these two different approaches and then applying them to specific patients can be challenging. Tools like the hematopoietic cell transplantspecific comorbidity index (HCT-CI) is a validated instrument that, when applied to patients with acute myeloid leukemia or myelodysplasia, was able to predict trends in survival when comparing myeloablative (MA-) to reduced-intensity (RI-) alloHCT, though the differences observed were not statistically significant $[16,17]$. While assessments like this can be helpful, in the end, these decisions often are based on the clinician's best judgment.

There are few reports in the literature where the question of MA- versus RI-alloHCT was addressed for MCL through direct comparison. Rodriguez and others at City of Hope performed a single-institution retrospective analysis of MA- versus RI-alloHCT in patients with non-Hodgkin lymphoma (NHL) [18]. In this 88-patient cohort, there were 15 patients with MCL: 10 received MA-alloHCT and 5 were treated with RI-alloHCT. Amongst these patients, 2-year OS and PFS were 30\% and 20\% (respectively) for RI-alloHCT compared to $50 \%$ and $40 \%$ (respectively) for MA-alloHCT. Despite the relative differences in these numbers, they were not statistically significant ( $\mathrm{P}=0.6$ for OS and 0.36 for PFS). However, the 2-year rate of relapse was higher in the RI-alloHCT group (60\%) compared to the MAalloHCT group $(20 \%)$, and this difference was significant $(\mathrm{P}=0.05)$. When the entire study cohort of NHL patients was evaluated, a similar pattern of findings was seen, with no difference seen in OS or PFS, but a higher rate of relapse after RI-alloHCT (28\%) versus 
MA-alloHCT $(12 \% ; \mathrm{P}=0.05)$. TRM is a greater concern with MAalloHCT, but when determined for the entire cohort, the difference between RI-alloHCT (28\%) and MAalloHCT $(38 \%)$ was not statistically significant $(\mathrm{P}=0.4)$; the authors did not report this measurement specifically for patients with MCL. Another smaller study from Germany performed a similar analysis but came to slightly different conclusions [19]: despite having only 25 total subjects with different types of lymphoma, they saw significantly better 2-year OS $(67 \%$ versus $23 \%$; $\mathrm{P}<0.02)$ and $\mathrm{EFS}(50 \%$ versus $23 \% ; \mathrm{P}=0.04)$ with $\mathrm{RI}$-alloHCT compared to MA-alloHCT, respectively. Non-relapse mortality (NRM) was also significantly different between the two groups, with a rate of $17 \%$ with RI-alloHCT compared to $54 \%$ following MA-alloHCT $(\mathrm{P}=0.03)$. This study contained only 2 patients with MCL, and they were both treated with RIalloHCT, so these differences could not be attributed to any particular feature of MCL. Given the relative lack of published evidence, it remains a challenge to determine when a myeloablative versus reduced-intensity approach is more appropriate for MCL. As stated above, using tools like the HCT-CI along with overall clinical impression probably remains the best strategy.

\section{Focus on RI-AlloHCT}

The majority of the published literature on alloHCT for MCL in the past 10 years has focused on RI-alloHCT. An effort to summarize a selection of these studies can be found in Table 1 [20-32]. Perhaps most importantly, similar to the study above by Tam and others, several of these trials included patients that had achieved long-term remission with this approach $[22,23,25,28]$. That said, there are significant limitations in these analyses. First, like many of the above studies, the total number of patients with MCL is generally very small, with larger groups of patients only seen in retrospective series. There also is a large amount of heterogeneity between the studies, likely reflecting institution-specific biases regarding preferred conditioning regimens, methods of GVHD prophylaxis, and prior treatments received. With rare exception, the patients included in these studies had relapsed or refractory disease; however, most studies included some patients that were in remission along with other patients with chemorefractory disease. There also was a variety of stem-cell sources (peripheral blood or bone marrow) and degree of HLA-matching between recipient and donor (matched versus mismatched, related versus unrelated). Finally, these studies only include highly-selected transplanted patients and cannot be used to compare outcomes with patients not receiving transplant. With an appreciation for these limitations, the following will discuss the general themes, as well as potentially interesting patterns, that emerge from a review of these data.

Like several studies mentioned above, success with RI-alloHCT seems to improve when this approach is used for patients with disease that is still chemosensitive. Unfortunately, the numbers of patients with MCL in these studies was not high enough to generate any statistically significant differences based on disease status at time of HCT, so these conclusions are drawn from trends in the data. For example, the series from Khouri and colleagues published in 2003 had some of the highest rates of overall and progression-free survival [26]. In this series, 8 of the 18 patients treated actually were in a complete remission (CR) at the time of HCT. Compare this to the results from Corradini and others published in 2007 [24]: only 1 of 17 patients with MCL examined in their analysis was in a $\mathrm{CR}$, and they saw among the lowest rates of overall and progression-free survival. Examined in a different way, Maris and coworkers noted in their report that the only pre-transplant feature that predicted for higher risk of relapse post-transplant was the number of prealloHCT treatments: 0 of 14 patients with less than 4 prior treatments relapsed, while relapse occurred in 4 of 19 patients with 4 or more pre-transplant treatments $(\mathrm{P}=0.01$ by univariate analysis) [27]. Similar findings were seen in several other studies when they were evaluated for in a heterogeneous population including multiple types of lymphoma [21, 25, 28, 29]. 
While these trends are suggestive, it should still be noted that these data do show that heavily-pretreated patients or patients with active disease can still potentially benefit from RI-alloHCT. In fact, a large retrospective study of hematologic malignancies treated with RI-alloHCT at the Fred Hutchinson Cancer Research Center (FHCRC) showed that the 16 patients with MCL in a CR had nearly identical rates of relapse per patient year (0.19) compared to the 25 patients that were not in a CR (0.20) [33].

Beyond status of disease at the time of RI-alloHCT, there were some additional predictors for success amongst patients with MCL. The study that addressed this most comprehensively was the report by Tam and colleagues from MDACC which was discussed previously [11]. The presence of chronic GVHD was associated with decreased rates of relapse (5\% at 6 years compared to $46 \%$ in patients without chronic GVHD; P $=0.05$ ). In a multivariate analysis, PFS for patients with MCL receiving RI-alloHCT was significantly better with the use of peripheral-blood stem cell grafts with best donor chimerism of at least $95 \%(\mathrm{P}=0.008)$ along with exposure to 4 or fewer prior therapies $(\mathrm{P}=0.02)$. However, disease status at time of transplant (i.e., CR versus refractory disease) did not predict for better disease control. The only factor that significantly improved OS on multivariate analysis was the absence of acute GVHD $(\mathrm{P}=0.02)$.

The retrospective study by Cook and others from the United Kingdom also sought to determine what other factors were associated with better outcomes [23]. While this study also showed findings favoring chemosensitive disease and disease in remission, they specifically addressed the use of alemtuzumab in the conditioning regimen. Patients who received alemtuzumab had a significantly better OS $(\mathrm{P}=0.027)$ and decreased NRM $(\mathrm{P}=$ 0.0017), with a trend toward better PFS $(P=0.075)$. However, relapse rates were higher after alemtuzumab, though not to a significant degree $(\mathrm{P}=0.3)$. They also reported relatively low rates of acute and chronic GVHD (see Table 1). The authors used this as evidence of a GVL effect, since the use of alemtuzumab in the conditioning regimen leads to in vivo $\mathrm{T}$ cell depletion, thus blunting an immune response against both lymphoma and host tissue, and (as will be discussed in more detail below) these relapses appeared salvageable with the use of donor lymphocyte infusion (DLI). The other study that included alemtuzumab in a substantial portion of patients was by Morris and colleagues [28]. While they did conclude that the use of this agent reduced the incidence of both acute and chronic GVHD compared to previously-reported rates (see Table 1), they did not speculate on its effect on lymphomaspecific outcomes.

Another facet of these studies that raises some additional questions is the use of further adoptive immunotherapeutic strategies to augment the response to RI-alloHCT. With the nearly-universal use of the anti-CD20 antibody, rituximab, in initial chemotherapy strategies for MCL, it would be expected that the vast majority of patients would have been exposed to this agent prior to HCT. A minority of the studies presented in this review included rituximab as part of the treatment strategy. One of these studies, by Khouri and others from 2003 [26], administered rituximab with the conditioning regimen in $72 \%$ of the patients in their study, and they did have some of the highest rates of overall and progression-free survival. However, as discussed above, this study included a relatively high number of patients in a CR which might bias the results toward better outcomes. Additionally, the authors did not report the results specifically for this subgroup of patients, so it is not clear how this affected outcomes even within their own study. Radioimmunotherapy (RIT) is another strategy where antibodies targeting antigens on the target tissue can deliver a radioisotope directly to the tumor to offer another anti-cancer effect [34]. Three studies have included this strategy as part of conditioning for RI-alloHCT, which include a total of 18 patients with MCL receiving yttrium-90 conjugated to the anti-CD20 antibody ibritumomab $[22,31,32]$. These patients in particular were rather heavily pre-treated, with all patients 
having received at least 2 chemotherapy regimens prior to the therapy on these studies. This, along with the small numbers of patients, makes it difficult to generate any conclusions about efficacy, but these strategies continue to be explored.

\section{Treatment of Post-AlloHCT Relapse of MCL}

Since the data available for alloHCT are relatively limited, it should come as no surprise that the data for management of relapsed MCL after alloHCT are even less robust. As most patients undergoing alloHCT have already received multiple lines of chemotherapy, disease is usually thought to be relatively chemorefractory at this stage. Since it remains a relatively unknown issue, such patients are probably best treated in the context of a clinical trial.

One interesting approach to this disease state is DLI. Some of the earliest reports of alloHCT for MCL included patients who relapsed and were shown to respond to DLI, including one patient that achieved durable CR that was approaching 4 years at the time of the report [7, 26]. Since that time, additional studies have paid specific attention to this phenomenon and reported responses to DLI in their relapsed patients, particularly the studies which included alemtuzumab as part of the pre-transplant conditioning. The report by Cook and colleagues included 36 relapses after RI-alloHCT, 32 of whom were further evaluable [23]. The 3-year OS amongst patients who received DLI for treatment of relapse was 60\% (with no deaths reported after approximately 15 months) compared to $0 \%$ for those who did not receive DLI for relapse $(P=0.006)$. Because of the improved outcomes seen with alemtuzumabcontaining conditioning and the significant responses seen with DLI, these authors advocated for a wider application of this strategy. This approach has not yet been validated in a prospective fashion, however. The study by Morris and others that included alemtuzumab reported the outcome of 2 patients with MCL that relapsed after RI-alloHCT and were given DLI, one of whom had a transient response [28]. DLI was also used in these studies to treat mixed chimerism, and both studies showed that this approach was able to help convert the majority of those recipients to complete donor chimerism. An important complication of DLI that needs to be considered prior to administration is GVHD. In the Morris study, the incidence of post-DLI GVHD was $28 \%$ (not segregated by histology), and in the Cook report, acute GVHD occurred in 11\% and chronic GVHD occurred in 37\% of recipients (all of whom had MCL).

Apart from the studies previously described, there have been three reports including patients with MCL where post-alloHCT relapse was the primary focus of the study. One study from the United Kindgom primarily addressed the role of DLI following the administration of chemotherapy [35]. Out of a total 17 patients described, 4 had MCL. Specific transplant conditioning regimens for these 4 patients were not described, though the majority of patients reported in this study received myeloablative conditioning and nearly all had received pretransplant alemtuzumab. Post-relapse chemotherapy included 2 cycles each of the following regimens: fludarabine, cyclophosphamide, and rituximab (FCR) for 2 patients; rituximab, ifosfamide, etoposide, and epirubicin (R-IVE) for 1 patient; and fludarabine, cytarabine, dexamethasone, cisplatin, and rituximab (FLUDAP-R) for 1 patient. Each patient then received DLI to a median of 2 doses (range 1-3). While the best response to chemotherapy was partial, 3 of the 4 patients achieved a CR following DLI. The $4^{\text {th }}$ patient (treated with FLUDAP-R) had progression of disease after DLI, but with additional therapy comprised of vincristine, dexamethasone, and radiotherapy, a late CR was achieved. All 4 patients developed GVHD as a consequence of DLI, which was the cause of death 12 months post-DLI for 1 patient. The remaining 3 patients were alive at a range of $13-40$ months post-DLI, though 1 patient (treated with FCR) was alive with relapsed disease 28 months after DLI. The second study that focused on post-alloHCT relapse included 72 patients at the University of Minnesota, 7 of whom had MCL [36]. Contrary to the other 
studies, the patients with MCL in this report did not receive DLI. The authors reported transplant conditioning regimens only for the entire cohort, but 24\% received MA-alloHCT while $76 \%$ received RI-alloHCT. Reduction of immunosuppression (RIS) as a lone intervention was able to induce a CR in 2 patients with MCL, further evidence of a GVL effect against MCL. Additionally, a subset of patients (which included 2 with MCL) treated with rituximab, alone or in combination with chemotherapy, showed an objective response. Of the 7 patients with MCL, 4 patients had died within 1 year following alloHCT, but the remaining 3 (43\%) were alive at 3 years post-alloHCT. In summary, these data for postalloHCT relapse show that the disease is potentially salvageable with either immune manipulation (DLI or RIS) or a re-challenge with anti-lymphoma chemotherapy.

Studies like the two mentioned above limit the analysis to patients who were candidates for and received a specific intervention. Instead, the following third study attempted to broadly describe outcome of post-alloHCT relapse. The group from FHCRC described 101 patients relapsing after RI-alloHCT that included 16 with MCL, with 11 (69\%) of these relapses occurring in those with a blastoid variant [37]. They identified two factors that predicted a worse outcome following post-RI-alloHCT relapse on multivariate analysis: relapse within 1 month of transplant (compared to > 6 months; $\mathrm{P}=0.004$ ) and aggressive NHL histology, which included blastoid MCL (compared to indolent NHL, which included classic MCL; P $=0.008$ ). Like the two studies above from the United Kingdom and Minnesota, this analysis also showed a potential to salvage relapses with a variety of interventions, including RIS alone, additional chemotherapy and/or radiation, and DLI. Even though 9 of the $16 \mathrm{MCL}$ patients responded to the initial postrelapse therapy, at the time of publication 11 of these patients had died, with a median postrelapse survival of 120 days (range 16-1515 days) (Gopal, et al., personal communication). More generally, the authors noted an additional finding that should lead clinicians treating such patients to pause: of the deaths seen following post-RI-alloHCT relapse, $40 \%$ were caused by treatment-related complications. These data underscore the need for such treatments to be offered with a measure of thought toward their potential adverse effects as well as their ultimate goals.

\section{Conclusions}

While substantial progress has been made in establishing a role for alloHCT in the management of MCL, many questions remain unanswered. Given the lack of prospective randomized trials, clinicians are left to rely on data from small prospective or larger retrospective studies. These studies in particular are fraught with selection bias. Clinicians are likely to offer an aggressive treatment such as this only to patients that they perceive will benefit most from it, thus excluding individuals with more comorbid medical conditions and sub-optimal performance status. And since these patients are offered this therapy preferentially, it is their outcomes that become part of these studies. Drop-outs and crossover to other therapies also occur frequently, so it is imperative that analyses be performed on an intention-to-treat basis to help account of these issues.

Until improvements in chemotherapy and/or autoHCT regimens can reliably produce longterm remissions for a large number of patients with MCL, alloHCT should be considered as a potential option for all who are afflicted with this disease. The emergence of RI-alloHCT has allowed this treatment to be offered to a larger number of patients, particularly for the older patients who are disproportionately affected by MCL. Preliminary data suggest that alloHCT is more successful when offered to patients either in remission or with chemosensitive disease. However, since life-threatening toxicity (particularly from GHVD) remains a serious concern from alloHCT, the appropriate time to offer this treatment during the natural history of this disease remains to be defined and likely is best individualized based on the biology of an individual's tumor, donor options, and patient preference. For 
those who undergo alloHCT and suffer a relapse, further immune manipulation with RIS or DLI may be able to achieve long-term disease control in a minority of patients. Future progress at addressing these challenges will likely require a focus on performing prospective randomized trials with collaboration between institutions or cooperative-groups to define the optimal role of alloHCT in MCL.

\section{Acknowledgments}

Funding provided by P01 CA04499 and gifts from Frank and Betty Vandermeer and Don and Debbie Hunkins. AKG is a Leukemia and Lymphoma Society Scholar in Clinical Research

Conflict of Interest Statement

Research support: Cephalon, Seattle Genetics, Spectrum, GlaxoSmithKline, Eli Lilly, Bio Marin, Abbott, Pfizer, Merck, and SBio.

Honoraria/Consulting: Millennium and Seattle Genetics.

\section{Literature Cited}

1. Dreyling M, Hiddemann W. Current treatment standards and emerging strategies in mantle cell lymphoma. Hematology Am Soc Hematol Educ Program. 2009:542-551. [PubMed: 20008239]

2. Geisler CH, Kolstad A, Laurell A, et al. Long-term progression-free survival of mantle cell lymphoma after intensive front-line immunochemotherapy with in vivo-purged stem cell rescue: a nonrandomized phase 2 multicenter study by the Nordic Lymphoma Group. Blood. 2008; 112:2687-2693. [PubMed: 18625886]

3. Dreyling M, Lenz G, Hoster E, et al. Early consolidation by myeloablative radiochemotherapy followed by autologous stem cell transplantation in first remission significantly prolongs progression-free survival in mantle-cell lymphoma: results of a prospective randomized trial of the European MCL Network. Blood. 2005; 105:2677-2684. [PubMed: 15591112]

4. Budde LE, Guthrie KA, Till BG, et al. Mantle cell lymphoma international prognostic index but not pretransplantation induction regimen predicts survival for patients with mantle-cell lymphoma receiving high-dose therapy and autologous stem-cell transplantation. J Clin Oncol. 2011; 29:30233029. [PubMed: 21730271]

5. Geisler CH, Kolstad A, Laurell A, et al. The Mantle Cell Lymphoma International Prognostic Index (MIPI) is superior to the International Prognostic Index (IPI) in predicting survival following intensive first-line immunochemotherapy and autologous stem cell transplantation (ASCT). Blood. 2010; 115:1530-1533. [PubMed: 20032504]

6. Budde LE, Guthrie KA, Till BG, et al. Prognostic factors on outcome of mantle cell lymphoma patients relapsing after autologous hematopoietic stem cell transplantation. Blood (ASH Annual Meeting Abstracts). 2009; 114 Abstract 3327.

7. Khouri IF, Lee MS, Romaguera J, et al. Allogeneic hematopoietic transplantation for mantle-cell lymphoma: molecular remissions and evidence of graft-versus-malignancy. Ann Oncol. 1999; 10:1293-1299. [PubMed: 10631455]

8. Zelentz AD, Abramson JA, Advani RA, et al. NCCN Clinical Practice Guidelines in Oncology: Non-Hodgkin's Lymphomas (version 4.2011). 2011

9. Khouri IF, Romaguera J, Kantarjian H, et al. Hyper-CVAD and high-dose methotrexate/cytarabine followed by stem-cell transplantation: an active regimen for aggressive mantle-cell lymphoma. J Clin Oncol. 1998; 16:3803-3809. [PubMed: 9850025]

10. Evens AM, Winter JN, Hou N, et al. A phase II clinical trial of intensive chemotherapy followed by consolidative stem cell transplant: long-term follow-up in newly diagnosed mantle cell lymphoma. Br J Haematol. 2008; 140:385-393. [PubMed: 18162124]

11. Tam CS, Bassett R, Ledesma C, et al. Mature results of the M.D. Anderson Cancer Center riskadapted transplantation strategy in mantle cell lymphoma. Blood. 2009; 113:4144-4152.

[PubMed: 19168784] 
12. Kasamon YL, Jones RJ, Diehl LF, et al. Outcomes of autologous and allogeneic blood or marrow transplantation for mantle cell lymphoma. Biol Blood Marrow Transplant. 2005; 11:39-46. [PubMed: 15625543]

13. Laudi N, Arora M, Burns L, et al. Efficacy of high-dose therapy and hematopoietic stem cell transplantation for mantle cell lymphoma. Am J Hematol. 2006; 81:519-524. [PubMed: 16755570]

14. Khouri IF, Keating M, Korbling M, et al. Transplant-lite: induction of graft-versus-malignancy using fludarabine-based nonablative chemotherapy and allogeneic blood progenitor-cell transplantation as treatment for lymphoid malignancies. J Clin Oncol. 1998; 16:2817-2824. [PubMed: 9704734]

15. McSweeney PA, Niederwieser D, Shizuru JA, et al. Hematopoietic cell transplantation in older patients with hematologic malignancies: replacing high-dose cytotoxic therapy with graft-versustumor effects. Blood. 2001; 97:3390-3400. [PubMed: 11369628]

16. Sorror ML, Maris MB, Storb R, et al. Hematopoietic cell transplantation (HCT)-specific comorbidity index: a new tool for risk assessment before allogeneic HCT. Blood. 2005; 106:29122919. [PubMed: 15994282]

17. Sorror ML, Sandmaier BM, Storer BE, et al. Comorbidity and disease status based risk stratification of outcomes among patients with acute myeloid leukemia or myelodysplasia receiving allogeneic hematopoietic cell transplantation. J Clin Oncol. 2007; 25:4246-4254. [PubMed: 17724349]

18. Rodriguez R, Nademanee A, Ruel N, et al. Comparison of reduced-intensity and conventional myeloablative regimens for allogeneic transplantation in non-Hodgkin's lymphoma. Biol Blood Marrow Transplant. 2006; 12:1326-1334. [PubMed: 17162215]

19. Bertz H, Illerhaus G, Veelken H, et al. Allogeneic hematopoetic stem-cell transplantation for patients with relapsed or refractory lymphomas: comparison of high-dose conventional conditioning versus fludarabine-based reduced-intensity regimens. Ann Oncol. 2002; 13:135-139. [PubMed: 11863095]

20. Armand P, Kim HT, Ho VT, et al. Allogeneic transplantation with reduced-intensity conditioning for Hodgkin and non-Hodgkin lymphoma: importance of histology for outcome. Biol Blood Marrow Transplant. 2008; 14:418-425. [PubMed: 18342784]

21. Baron F, Storb R, Storer BE, et al. Factors associated with outcomes in allogeneic hematopoietic cell transplantation with nonmyeloablative conditioning after failed myeloablative hematopoietic cell transplantation. J Clin Oncol. 2006; 24:4150-4157. [PubMed: 16896000]

22. Bethge WA, Lange T, Meisner C, et al. Radioimmunotherapy with yttrium-90-ibritumomab tiuxetan as part of a reduced- intensity conditioning regimen for allogeneic hematopoietic cell transplantation in patients with advanced non-Hodgkin lymphoma: results of a phase 2 study. Blood. 2010; 116:1795-1802. [PubMed: 20530284]

23. Cook G, Smith GM, Kirkland K, et al. Outcome following Reduced-Intensity Allogeneic Stem Cell Transplantation (RIC AlloSCT) for relapsed and refractory mantle cell lymphoma (MCL): a study of the British Society for Blood and Marrow Transplantation. Biol Blood Marrow Transplant. 2010; 16:1419-1427. [PubMed: 20399879]

24. Corradini P, Dodero A, Farina L, et al. Allogeneic stem cell transplantation following reducedintensity conditioning can induce durable clinical and molecular remissions in relapsed lymphomas: pre-transplant disease status and histotype heavily influence outcome. Leukemia. 2007; 21:2316-2323. [PubMed: 17597807]

25. Hertzberg M, Grigg A, Gottlieb D, et al. Reduced-intensity allogeneic haemopoietic stem cell transplantation induces durable responses in patients with chronic B-lymphoproliferative disorders. Bone Marrow Transplant. 2006; 37:923-928. [PubMed: 16565735]

26. Khouri IF, Lee MS, Saliba RM, et al. Nonablative allogeneic stem-cell transplantation for advanced/recurrent mantle-cell lymphoma. J Clin Oncol. 2003; 21:4407-4412. [PubMed: 14645431]

27. Maris MB, Sandmaier BM, Storer BE, et al. Allogeneic hematopoietic cell transplantation after fludarabine and $2 \mathrm{~Gy}$ total body irradiation for relapsed and refractory mantle cell lymphoma. Blood. 2004; 104:3535-3542. [PubMed: 15304387] 
28. Morris E, Thomson K, Craddock C, et al. Outcomes after alemtuzumab-containing reducedintensity allogeneic transplantation regimen for relapsed and refractory non-Hodgkin lymphoma. Blood. 2004; 104:3865-3871. [PubMed: 15304395]

29. Robinson SP, Goldstone AH, Mackinnon S, et al. Chemoresistant or aggressive lymphoma predicts for a poor outcome following reduced-intensity allogeneic progenitor cell transplantation: an analysis from the Lymphoma Working Party of the European Group for Blood and Bone Marrow Transplantation. Blood. 2002; 100:4310-4316. [PubMed: 12393626]

30. Shea T, Johnson J, Westervelt P, et al. Reduced-Intensity Allogeneic Transplantation Provides High Event-Free and Overall Survival in Patients with Advanced Indolent B Cell Malignancies: CALGB 109901. Biol Blood Marrow Transplant. 2011; 17:1395-1403. [PubMed: 21296675]

31. Shimoni A, Zwas ST, Oksman Y, et al. Ibritumomab tiuxetan (Zevalin) combined with reducedintensity conditioning and allogeneic stem-cell transplantation (SCT) in patients with chemorefractory non-Hodgkin's lymphoma. Bone Marrow Transplant. 2008; 41:355-361. [PubMed: 18026153]

32. Gopal AK, Guthrie KA, Rajendran J, et al. Y-Ibritumomab tiuxetan, fludarabine, and TBI-based nonmyeloablative allogeneic transplantation conditioning for patients with persistent high-risk Bcell lymphoma. Blood. 2011; 118:1132-1139. [PubMed: 21508413]

33. Kahl C, Storer BE, Sandmaier BM, et al. Relapse risk in patients with malignant diseases given allogeneic hematopoietic cell transplantation after nonmyeloablative conditioning. Blood. 2007; 110:2744-2748. [PubMed: 17595333]

34. Zhang MM, Gopal AK. Radioimmunotherapy-based conditioning regimens for stem cell transplantation. Semin Hematol. 2008; 45:118-125. [PubMed: 18381107]

35. Russell NH, Byrne JL, Faulkner RD, et al. Donor lymphocyte infusions can result in sustained remissions in patients with residual or relapsed lymphoid malignancy following allogeneic haemopoietic stem cell transplantation. Bone Marrow Transplant. 2005; 36:437-441. [PubMed: 15980879]

36. Wudhikarn K, Brunstein CG, Bachanova V, et al. Relapse of Lymphoma after Allogeneic Hematopoietic Cell Transplantation: Management Strategies and Outcome. Biol Blood Marrow Transplant. 2011; 17:1497-1504. [PubMed: 21338707]

37. Ram R, Gooley TA, Maloney DG, et al. Histology and time to progression predict survival for lymphoma recurring after reduced-intensity conditioning and allogeneic hematopoietic cell transplantation. Biol Blood Marrow Transplant. 2011; 17:1537-1545. [PubMed: 21536145] 


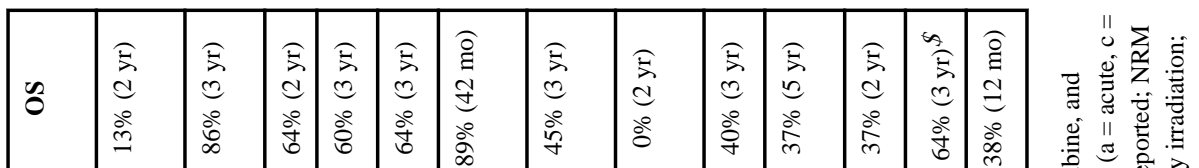

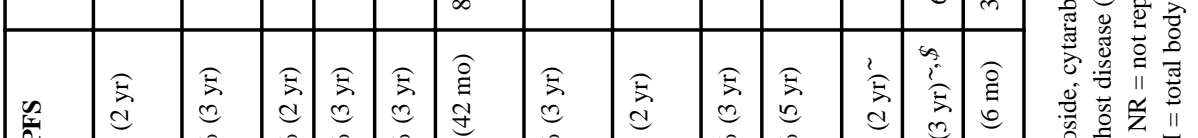

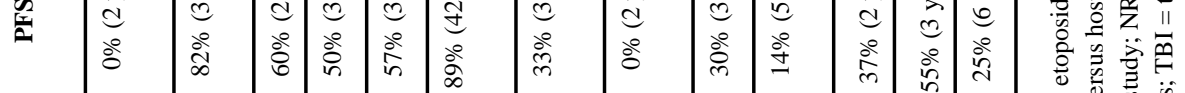

\begin{tabular}{|c|c|c|c|c|c|c|c|c|c|c|c|c|c|}
\hline & & & & & & & & & & & & & \\
\hline 竞 & 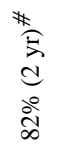 & $\begin{array}{l}\hat{\Xi} \\
\stackrel{0}{\circ} \\
\stackrel{0}{=}\end{array}$ & $\begin{array}{l}\widehat{E} \\
\text { d } \\
\text { o } \\
\text { dे }\end{array}$ & 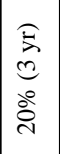 & 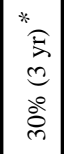 & 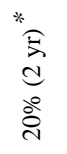 & $\begin{array}{l}\hat{E} \\
\tilde{C} \\
\therefore \\
\tilde{N}\end{array}$ & 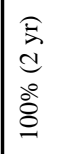 & $\begin{array}{l}\hat{E} \\
0 \\
0 \\
0 \\
\text { co }\end{array}$ & 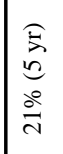 & 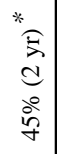 & 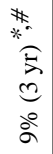 & 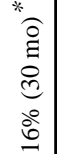 \\
\hline & & & & $*$ & & & & & & & & & \\
\hline
\end{tabular}

$\hat{2}$

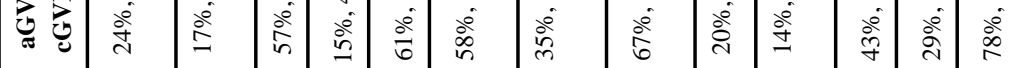

\begin{tabular}{|c|c|c|c|c|c|c|c|c|c|c|c|c|}
\hline & & & & & & & & & & & & \\
\hline Tू & ڤ̆ & $\begin{array}{l}\text { ह } \\
\text { ㅇ }\end{array}$ & $\begin{array}{l}\text { ఏ } \\
\text { הે }\end{array}$ & $\begin{array}{l}\text { ఏ } \\
\text { D }\end{array}$ & 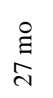 & 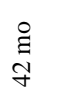 & 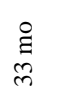 & 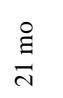 & $\begin{array}{l}\stackrel{g}{\Xi} \\
\text { ¿ }\end{array}$ & 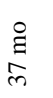 & $\begin{array}{l}\stackrel{̊}{E} \\
\text { స̃ }\end{array}$ & $\begin{array}{l}\stackrel{g}{g} \\
\text { in }\end{array}$ \\
\hline
\end{tabular}

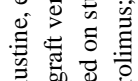

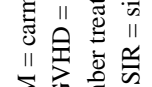

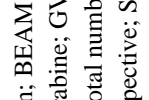
.

苍 这

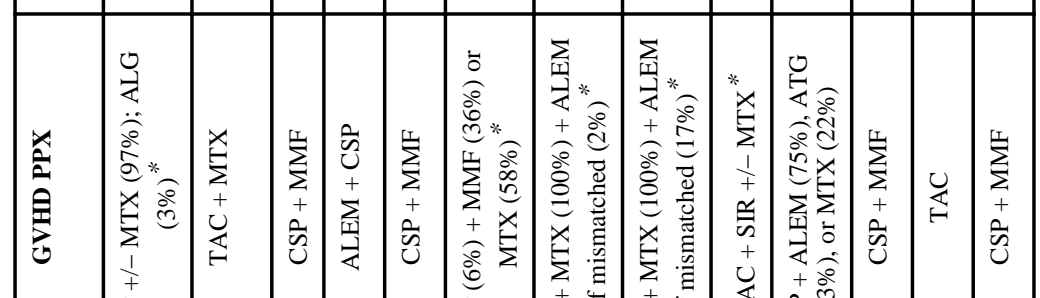

\begin{tabular}{|c|c|c|c|c|c|c|c|c|c|c|c|c|c|}
\hline & $\begin{array}{l}+ \\
\stackrel{7}{7}\end{array}$ & & & & & 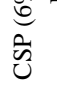 & $\begin{array}{l}\vec{z} \\
+ \\
\overrightarrow{\tilde{U}}\end{array}$ & 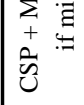 & $\stackrel{\mathbb{E}}{E}$ & $\begin{array}{l}++s \\
\tilde{v}^{2} \\
\tilde{v}\end{array}$ & & & \\
\hline 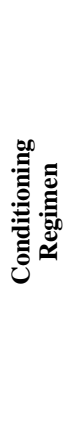 & 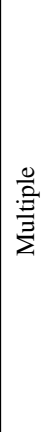 & 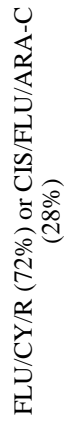 & 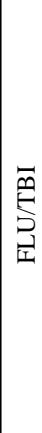 & 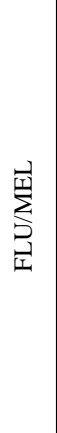 & 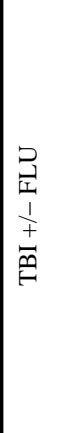 & 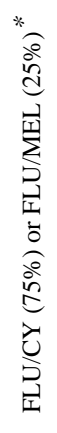 & 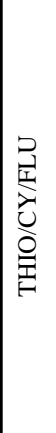 & \begin{tabular}{ll}
\multicolumn{5}{c}{} \\
0 \\
0 \\
0 \\
0 \\
0
\end{tabular} & 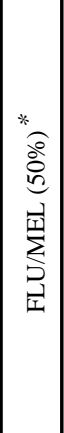 & 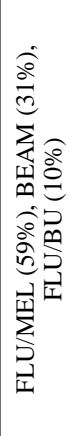 & 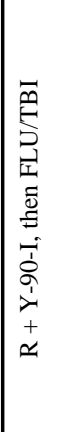 & 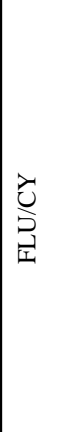 & 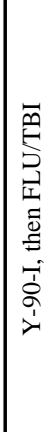 \\
\hline 胥总 & 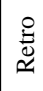 & $\stackrel{8}{2}$ & $\stackrel{8}{2}$ & 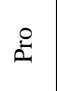 & $\frac{8}{\mathscr{Q}}$ & 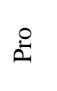 & 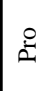 & 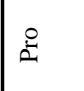 & $\frac{\mathscr{V}}{\mathscr{e}}$ & $\stackrel{\circ}{\dddot{E}}$ & $\stackrel{2}{2}$ & $\approx$ & 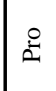 \\
\hline $\mathrm{z}$ & 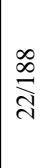 & $\stackrel{\infty}{\infty}$ & लెल & $\begin{array}{l}\infty \\
\stackrel{\infty}{\Xi}\end{array}$ & 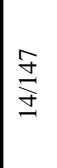 & $\stackrel{\circ}{\check{\sigma}}$ & $\begin{array}{l}8 \\
\frac{8}{3}\end{array}$ & $\frac{1}{d}$ & $\begin{array}{l}\frac{\hat{\infty}}{n} \\
\underline{n}\end{array}$ & $\frac{8}{2}$ & $\frac{\mathfrak{q}}{\infty}$ & $\frac{\text { 竎 }}{7}$ & $\frac{9}{\infty}$ \\
\hline 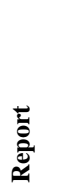 & 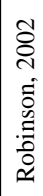 & 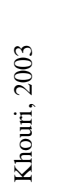 & 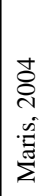 & 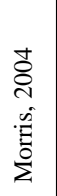 & 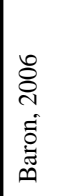 & 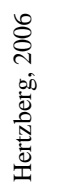 & 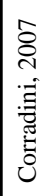 & 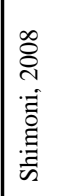 & 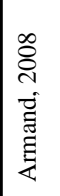 & $\begin{array}{l}\text { 을 } \\
\text { ì } \\
\text { it } \\
0\end{array}$ & 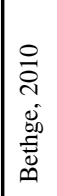 & 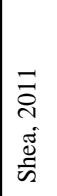 & 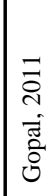 \\
\hline
\end{tabular}




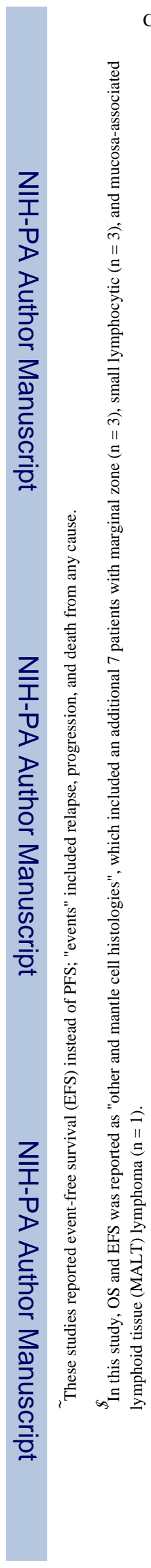

